\title{
Numerical Modelling of Coupled Rooms: Evaluation of Decay Times via Method Employing Hilbert Transform
}

\author{
M. MEISSNER \\ Institute of Fundamental Technological Research, Polish Academy of Sciences
}

A. Pawińskiego 5B, 02-106 Warsaw, Poland

\begin{abstract}
In the paper, a usefulness of the Hilbert transform in a computer modelling of reverberant behaviour of rooms is demonstrated for the enclosure consisting of two coupled subrooms. In numerical simulations a decay of the sound pressure is computed and the Hilbert transform methodology is used to determine an envelope of this decay. Calculation results have shown that, because of the mode localization, a distribution of absorbing material has a great influence on decay times evaluated from changes in the pressure envelope. For example, when a difference between the sound damping in subrooms is large, the localization effect is responsible for a creation of a nonlinear decay of the sound pressure level characterized by rapid early and slow late sound decays.
\end{abstract}

PACS: 43.55.Br, 43.55.Ka, 43.20.Ks, 43.60.Ac

\section{Introduction}

The Hilbert transform has been recognized as very important method for the signal analysis in different branches of science and technology [1-3]. In a numerical modelling of the sound decay in enclosures, the Hilbert transform allows to construct the analytic signal and to calculate the envelope of decaying acoustic signal. It is of special importance for the evaluation of decay times in coupled rooms which yield usually a nonlinear profile of the pressure level decay [4-7]. However, an application of the Hilbert transform in sound decay calculations gives meaningful numerical data only for restrictive class of enclosures [8]. The accurate results are obtained for room systems with relatively small sound damping because in this case the product of exponentially decaying signal and a harmonic signal (sine or cosine function) satisfies approximately the Bedrosian identity [9].

The paper is organized as follows. In the first part the most important properties of the Hilbert transform are shortly discussed. Then, a utility of the Hilbert transform is examined using sound decay simulations performed for the enclosure consisting of two connected rectangular subrooms.

\section{Hilbert transform and analytic signal representation}

In the time domain, the Hilbert transform $\mathcal{H}$ of a real-valued signal $x(t)$, which is denoted by $\hat{x}(t)$, is a convolution between $x(t)$ and the Hilbert transformer $1 / \pi t$, thus it is defined as

$$
\mathcal{H}[x(t)]=\hat{x}(t)=x(t) * \frac{1}{\pi t}=\frac{1}{\pi} \mathrm{P} \int_{-\infty}^{\infty} \frac{x(\tau)}{t-\tau} \mathrm{d} \tau,
$$

where $\mathrm{P}$ in the front of integral indicates the Cauchy principal value. The Hilbert transform has been widely used in physics, engineering and mathematics because it is the basic mathematical tool for a direct examination of instantaneous properties of signals. The real signal is usually represented by the amplitude and the frequency according to its physical attributes. The classical method for a determination of signal characteristics is to add $\hat{x}(t)$ as the imaginary part to $x(t)$ to produce the so-called analytic signal

$$
z(t)=x(t)+\mathrm{j} \hat{x}(t)=A(t) \mathrm{e}^{\mathrm{j} \psi(t)},
$$

and then extract $A(t)=\sqrt{x^{2}(t)+\hat{x}^{2}(t)}$ and $\psi(t)=$ $\tan ^{-1}(\hat{x}(t) / x(t))$ as its instantaneous amplitude (envelope, magnitude) and phase. The frequency $\omega(t)$ of the analytic signal $z(t)$ is defined as: $\omega(t)=\mathrm{d} \psi_{\mathrm{u}} / \mathrm{d} t$, where $\psi_{\mathrm{u}}(t)$ is the continuous, unwrapped phase, that is

$$
\psi_{\mathrm{u}}(t)=\psi(t)+\Gamma(t),
$$

where $\Gamma(t)$ is an integer multiple of $\pi$-valued function designed to insure a continuous phase function. The Hilbert transform has been found very useful for many types of signals, especially for amplitude modulated ones, modelled as a product of two functions. Under certain conditions, the simple method for computing such a product was found by Bedrosian [9]. If two functions, say $x(t)$ and $y(t)$, represent signals with non-overlapping spectra and the spectrum of $x(t)$ is lower than that of $y(t)$, then 


$$
\mathcal{H}[x(t) y(t)]=x(t) \mathcal{H}[y(t)],
$$

thus, to compute the Hilbert transform of the product of a low-pass signal with a high-pass signal, only the high-pass signal needs to be transformed. This result is known in the literature as the Bedrosian theorem and the formula (4) is called the Bedrosian identity.

\section{Numerical simulation of reverberant sound decay}

In order to demonstrate a utility of the Hilbert transform technique in a prediction of reverberant behaviour of rooms, a numerical simulation of the sound decay was carried out for a room system consisting of two coupled rectangular subrooms (Fig. 1). The subrooms have the height $h$ of $3 \mathrm{~m}$ and their lengths and widths are the following: $l_{1}=4 \mathrm{~m}, l_{2}=5.7 \mathrm{~m}, w_{1}=5 \mathrm{~m}$ and $w_{2}=8 \mathrm{~m}$. The acoustic coupling between subrooms is realized by the opening having the height $h$, the width $w$ of $2 \mathrm{~m}$ and the thickness $d$ of $0.3 \mathrm{~m}$. The room is excited by the harmonic point source located in subroom $B$ at the position: $x=2 \mathrm{~m}, y=5 \mathrm{~m}, z=1 \mathrm{~m}$.

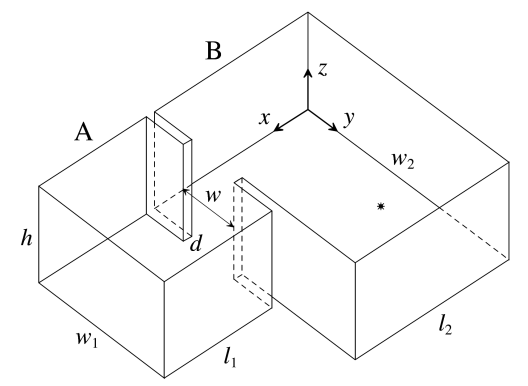

Fig. 1. Irregularly shaped enclosure consisting of two connected rectangular subrooms denoted by $A$ and $B$. Symbol indicates position of sound source.

In the case of rooms with a complex geometry the first step towards determining the room response is a computation of the eigenfunctions $\Phi_{m}$ and the modal frequencies $\omega_{m}(m=1,2,3, \ldots)$. Since lightly damped rooms are considered, the functions $\Phi_{m}$ can be well approximated by eigenfunctions computed for rigid room walls [10]. Thus, one can write

$$
\Phi_{m}(\boldsymbol{r})=\Psi_{\kappa}(x, y) \Theta_{\nu}(z),
$$

where $\kappa=1,2,3, \ldots, \nu=0,1,2, \ldots, \boldsymbol{r}=(x, y, z)$ is a position vector, $\Theta_{0}=1 / \sqrt{h}, \Theta_{\nu}=\sqrt{2 / h} \cos (\pi \nu z / h)$ for $\nu>0$, and the eigenfunctions $\Psi_{\kappa}$ are normalized over a horizontal cross-section of the room. In this case, the modal frequencies are given by

$$
\omega_{m}=\sqrt{\omega_{\kappa}^{2}+(\pi \nu c / h)^{2}},
$$

where $\omega_{\kappa}$ is an eigenfrequency for the function $\Psi_{\kappa}$. Spatial distributions of eigenfunctions $\Psi_{\kappa}$ in $(x, y)$ plane and the frequencies $\omega_{\kappa}$ were calculated with the aid of numerical implementation based on the forced oscillator method with the finite difference algorithm [11]. Shapes of the function $\Psi_{\kappa}$ for certain modes are plotted in Fig. 2. These data show that for some modes the acoustic energy is distributed quite regularly inside the analysed room system (Fig. 2b,d), however there are also such modes for which the acoustic energy is concentrated within the one of subrooms (Fig. 2a,c). The phenomenon of a modal energy accumulation in some parts of enclosed spaces is characteristic for coupled rooms and enclosures having an irregular geometry, and it is called the mode localization.

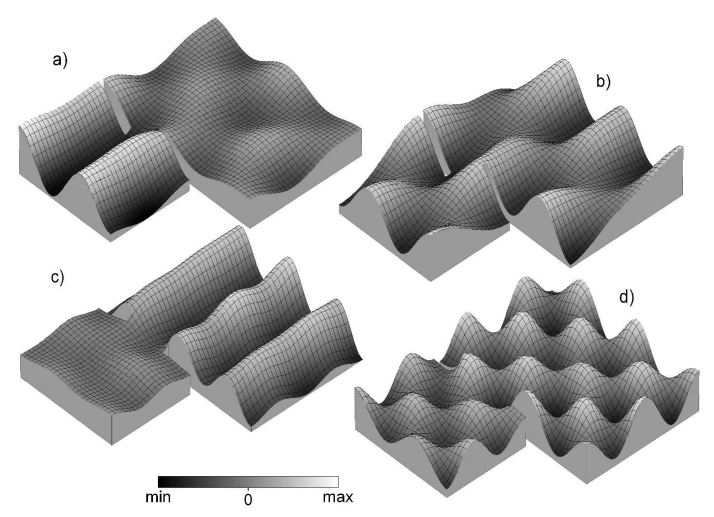

Fig. 2. Shapes of eigenfunctions $\Psi_{\kappa}$ for mode number $m:$ (a) 40, (b) 48, (c) 73, (d) 123 .

In the numerical simulation it is assumed that walls of subrooms $A$ and $B$ are covered by absorbing materials providing a relatively small sound damping and the random-absorption coefficients $\alpha_{a}$ and $\alpha_{b}$ characterize damping properties of these materials. To ensure a constant value of the Schroeder frequency [12] and to examine the effect of absorbing material distribution on the room response, values of coefficients $\alpha_{a}$ and $\alpha_{b}$ were selected in such a way that the total absorption $\mathcal{A}$ in subrooms remained constant, that is

$$
\mathcal{A}=\bar{\alpha}\left(S_{a}+S_{b}\right)=\alpha_{a} S_{a}+\alpha_{b} S_{b}=\text { const },
$$

where $\bar{\alpha}$ is the mean value of absorption coefficient and $S_{a}$ and $S_{b}$ are surfaces of walls in subrooms $A$ and $B$, respectively. For the sake of model simplicity the wall impedance $Z$ was assumed to be purely real, i.e. the mass and stiffness of the absorbing material are neglected. This corresponds to the damping of a sound wave on the wall with no phase change upon reflection. For a given value of the absorption coefficient $\bar{\alpha}$, the wall impedances on subrooms walls were found from the well-known relationship between the random-absorption coefficient $\alpha$ and the impedance ratio $\xi[13]$ :

$$
\alpha=\frac{8}{\xi}\left[1+\frac{1}{1+\xi}-\frac{2}{\xi} \ln (1+\xi)\right], \quad \xi=Z / \rho c .
$$

Computations were performed for the coefficient $\bar{\alpha}$ equal to 0.09 . It is easy to calculate that the Schroeder frequency [12], corresponding to this value of $\bar{\alpha}$, is $f_{s}=$ $174.2 \mathrm{~Hz}$. Below this frequency 150 eigenmodes were found. For this set of modes a sound decay in an ob- 
servation point located in subroom $A$ at the position: $x=8 \mathrm{~m}, y=2 \mathrm{~m}, z=1.8 \mathrm{~m}$, was simulated. The effect of non-exponential sound decay was characterized by two metrics: the early decay time (EDT) predicted from the pressure level drop from 0 to $-10 \mathrm{~dB}$ and late reverberation time (LDT) estimated from a decrease in this level from -50 to $-60 \mathrm{~dB}$.

Results of a numerical simulation, presented in Fig. 3, were obtained for a material with the absorption coefficient of 0.09 uniformly distributed on subrooms' walls and a source frequency of $128 \mathrm{~Hz}$ corresponding approximately to the frequency of mode 73 . The eigenfunction $\Psi_{\kappa}$ for mode 73 is shown in Fig. 2c, thus this mode is strongly localized in subroom $B$. The data in Fig. 3 show a temporal decay of the normalized sound pressure $p / p_{\max }$ and changes in the envelope $L_{E}$ of pressure level in the time interval corresponding approximately to the reverberation time. The pressure amplitude was determined with an application of the Hilbert transform. Due to an influence of several modes on the room response, the pressure amplitude begins to fluctuate in the initial stage of sound decay and it corresponds to irregular variations in the envelope $L_{E}$ for the time $t$ smaller than one second. Despite these changes, a mean trend in a decrease of the envelope $L_{E}$, evaluated by the regression method, is well reproduced by the fit curve corresponding approximately to a straight line as indicated by the values of decay time in early and late stage of sound decay.
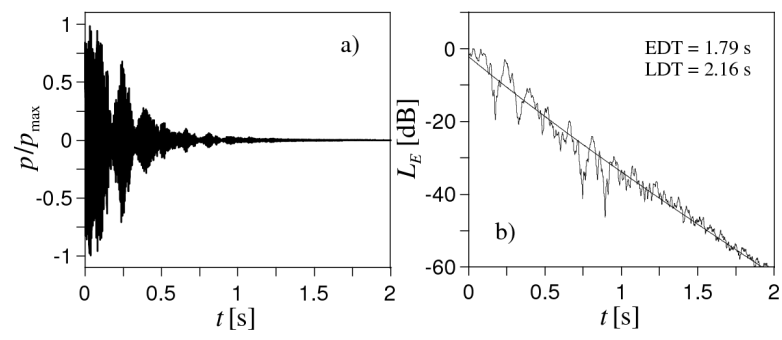

Fig. 3. (a) Normalized sound pressure $p / p_{\max }$ and (b) envelope $L_{E}$ of pressure level versus time $t$ for source frequency of $128 \mathrm{~Hz}$ and absorption coefficients $\alpha_{a}=$ $\alpha_{b}=0.09$. Solid smooth line denotes best-fit curve calculated by polynomial regression.

A contribution of individual modes in the room response depends on both the modal amplitude $A_{m}$ and the modal reverberation time $T_{m}$. From Ref. [14] it results that the expression for $A_{m}$ can be written as

$$
A_{m}=\frac{Q_{m} \omega_{m} \Phi_{m}(\boldsymbol{r})}{\sqrt{\left(\omega_{m}^{2}-r_{m}^{2}\right)\left[\left(\omega_{m}^{2}-\omega^{2}\right)^{2}+4 r_{m}^{2} \omega^{2}\right]}},
$$

thus it depends not only on the source frequency $\omega$ but also on the source location (through the parameter $Q_{m}$ ), the distribution of eigenfunction, the position of an observation point and the wall impedance (through the damping coefficient $r_{m}$ ). The bar charts in Fig. 4 show dependences of the normalized modal amplitude $\left|A_{m}\right| / A_{\max }$ and the modal reverberation time $T_{m}$ on the mode num- ber $m$ for previously assumed: the source frequency and values of the absorption coefficients $\alpha_{a}$ and $\alpha_{b}$. The amplitude $A_{\max }$ corresponds to the maximum value of $\left|A_{m}\right|$ in the considered set of eigenmodes. As is evident from Fig. 4a, for the frequency of $128 \mathrm{~Hz}$ two neighbouring modes (modes 72 and 73) are dominant in the room response. Thus, the observed changes in the envelope $L_{E}$ are due to the fact that the pressure amplitude begins to oscillate with a frequency equal to the difference between frequencies of neighbouring modes (beating effect). In turn, the data in Fig. 4b show that despite identical values of the absorption coefficients $\alpha_{a}$ and $\alpha_{b}$, the modal reverberation time $T_{m}$ assumes significantly different values. For modes 72 and 73 it equals $1.32 \mathrm{~s}$ and $2.16 \mathrm{~s}$. It results in a visible disagreement between evaluated decay times (Fig. 3b) because in the initial stage of sound decay the dominant mode with a smaller reverberation time affects the room response.
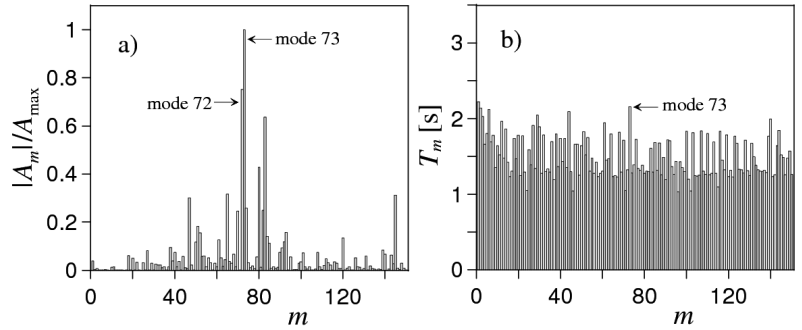

Fig. 4. (a) Normalized modal amplitude $\left|A_{m}\right| / A_{\max }$ and (b) modal reverberation time $T_{m}$ versus mode number $m$ for source frequency of $128 \mathrm{~Hz}$ and absorption coefficients $\alpha_{a}=\alpha_{b}=0.09$.
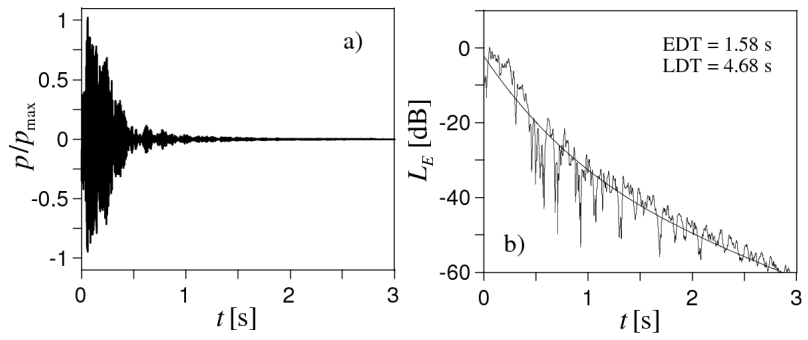

Fig. 5. (a) Normalized sound pressure $p / p_{\max }$ and (b) envelope $L_{E}$ of pressure level versus time $t$ for source frequency of $133 \mathrm{~Hz}$ and absorption coefficients $\alpha_{a}=$ 0.185 and $\alpha_{b}=0.04$. Solid smooth line denotes best-fit curve calculated by polynomial regression.

In the second numerical example, the source frequency was shifted to $133 \mathrm{~Hz}$ and the absorption coefficients $\alpha_{a}$ and $\alpha_{b}$ were equal to 0.185 and 0.04 , respectively. It means that subroom $A$ provides much more greater sound damping than subroom $B$. Simulation data in Fig. 5a illustrate temporal changes in the sound pressure in this case and the interesting thing to note is that the sound pressure rapidly decreases in the initial stage of reverberation process. This results in the nonlinear decay of the 

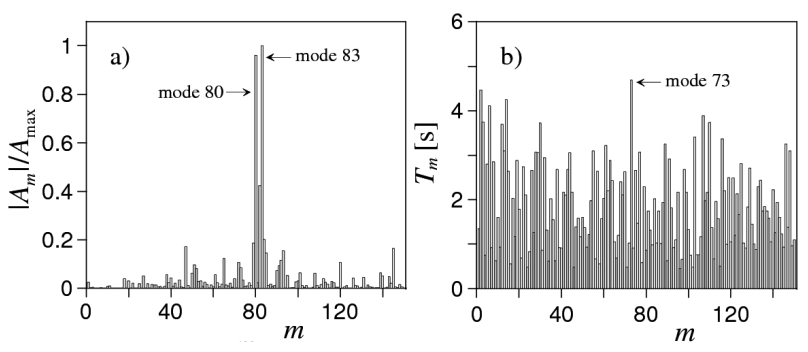

Fig. 6. (a) Normalized modal amplitude $\left|A_{m}\right| / A_{\max }$ and (b) modal reverberation time $T_{m}$ versus mode number $m$ for source frequency of $133 \mathrm{~Hz}$ and absorption coefficients $\alpha_{a}=0.185$ and $\alpha_{b}=0.04$.

pressure level because its envelope $L_{E}$ exhibits the "sagging" appearance with the fast initial decay and the visibly slower late decay (Fig. 5b). It is reflected in the decay times estimated from a slope of best fitting curve because the early decay time is found to be almost three times smaller than the late decay time. An explanation of such behaviour of the sound decay may be sought through analysis of numerical data in Fig. 6. When the source frequency is shifted to $133 \mathrm{~Hz}$, two modes (modes 80 and 83) of slightly different amplitudes are recognised to be dominant. However, modal reverberation times corresponding to these modes are so small that they vanish in the initial stage of decay. Therefore, in the late stage of sound decay the mode 73 with a long modal reverberation time becomes predominant, resulting in the creation of nonlinear profile of the decay curve.

\section{Conclusions}

A usefulness of the Hilbert transform in a computer modelling of the reverberation phenomenon was examined using sound decay simulations performed for the enclosure consisting of two connected rectangular subrooms. In a theoretical model, the room response was described in terms of its normal eigenmodes. Spatial distributions of modes and corresponding eigenfrequencies were computed using a numerical procedure based on the forced oscillator method. It was found that in the analysed enclosure there are some modes for which the acoustic energy is concentrated within the one of subrooms. This phenomenon of modal energy accumulation is characteristic for coupled rooms and is called the mode localization.

Calculation results revealed that the Hilbert transform is a useful tool for smoothing a profile of decaying pressure level before an application of the regression method for an estimation of decay times. As was shown, this is very important for irregular sound decays occurring, for example, for the decay deformed by fluctuations because of two significant modes close in the frequency (beating effect). Because of the mode localization a distribution of the absorbing material has a great influence on the reverberant behaviour of coupled rooms. In the case of a large difference between the sound damping inside subrooms, this effect is responsible for a creation of a nonlinear decay of the sound pressure level characterized by the rapid early and slow late sound decays.

\section{References}

[1] G. Buresti, G. Lombardi, J. Bellazzini, Chaos, Solit. Frac. 20, 149 (2004).

[2] Y. Yan, K. Ahmad, M. Kunduk, D. Bless, J. Voice 19, 161 (2005).

[3] L. Huageng, F. Xingjie, E. Bugra, Am. Inst. Aero. Astr. J. 47, 923 (2009).

[4] M. Meissner, Arch. Acoust. 32, 213 (2007).

[5] M. Meissner, in: Renewable Energy, Innovative Technologies and New Ideas, Eds. D. Chwieduk, R. Domański, M. Jaworski, Publishing House of Warsaw University of Technology, Warsaw 2008, p. 265.

[6] M. Meissner, Arch. Acoust. 33, 159 (2008).

[7] M. Meissner, Acta Phys. Pol. A 118, 123 (2010).

[8] M. Meissner, Arch. Acoust. 34, 491 (2009).

[9] E. Bedrosian, Proc. IEEE 51, 868 (1963).

[10] E. Dowell, G. Gorman, D. Smith, J. Sound Vib. 52, 519 (1977).

[11] M. Meissner, Applied Acoust. 69, 583 (2008).

[12] M. Schroeder, J. Acoust. Soc. Am. 99, 3240 (1996).

[13] L.E. Kinsler, A.R. Frey, Fundamentals of Acoustics, Wiley, New York 1962.

[14] M. Meissner, Cent. Eur. J. Phys. 5, 293 (2007). 